\title{
A Multihypothesis Sequential Probability Test for Fault Detection and Identification of Vehicles' Ultrasonic Parking Sensors
}

\author{
Mamoun F. Abdel-Hafez \\ Department of Mechanical Engineering, American University of Sharjah, P.O. Box 26666, Sharjah, UAE \\ Correspondence should be addressed to Mamoun F. Abdel-Hafez, mabdelhafez@aus.edu
}

Received 28 April 2011; Revised 30 July 2011; Accepted 3 October 2011

Academic Editor: Shaojun Feng

Copyright (๑) 2011 Mamoun F. Abdel-Hafez. This is an open access article distributed under the Creative Commons Attribution License, which permits unrestricted use, distribution, and reproduction in any medium, provided the original work is properly cited.

\begin{abstract}
This paper presents a sequential fault detection and identification algorithm for detecting a fault in a vehicle's ultrasonic parking sensors. The algorithm identifies a bias fault in any of the ultrasonic sensors by computing the probability of having that bias fault given a carefully constructed measurement residual that is only a function of the measurement noise and the possible measurement fault. A set of bias hypotheses is assumed and initially given equal alarm probability. It is assumed that only one sensor will acquire a bias at any given time. Once the probability of a hypothesis approaches 1, that hypothesis is declared as the correct hypothesis and the bias associated with the hypothesis is removed from the sensors' reading. The accuracy and convergence characteristics of the proposed algorithm are verified using experimental results. This study is essential to ensure accurate operation of vehicle's ultrasonic parking sensors.
\end{abstract}

\section{Introduction}

The problem of a fault occurring in dynamic systems is being addressed continuously by researchers due to its high impact on the accuracy and integrity of the overall system performance [1-17]. Among this effort, extensive study is focused on the integrity monitoring of navigation systems [1-5]. Other researchers tackled the signal processing and integrity monitoring of sonar sensors [6-10]. Also, researchers address the integrity monitoring and fault detection of automotive sensors and systems [11-18].

A sensor fault can be modeled in two ways first, as an additive random process with an associated statistics, that is, mean and covariance [1-3]. Alternatively, other researchers account for the fault as an increase in the magnitude of the measurements noise covariance $[4,5]$. The change in the measurement noise covariance is detected using various statistical algorithms. The problem of change detection was formulated by reference [19] for independent observations with a given prior probability density distribution. Upon the onset of a fault, the observations' probability density will change to another distribution that needs to be identified.
In transport vehicles, new sensors are constantly being added to enhance passengers comfort and increase vehicle's safety $[11,12]$. With the addition of new sensors, it is imperative to continuously check for the integrity of the sensors' readings to completely and correctly achieve their given tasks [13-18].

Model-based techniques have been used in the literature to check for automotive sensors faults [20]. In these techniques, different measurement models are tested in a probabilistic manner to identify possible faults in the vehicle sensors $[21,22]$. Information-theoretic stopping rules were presented in references $[19,20]$ for efficient sequential multihypothesis testing and fault detection of abrupt changes in stochastic systems.

Ultrasonic sensors are currently being used in most vehicles to assist drivers in parking their vehicles by identifying the range from the perimeters of the vehicle to the surrounding vehicles, walls, or objects. Although, this feature is very helpful in preventing accidental pumping of the vehicle into the surrounding bodies, a fault in one or more of these sensors may cause adverse results. Therefore, the quality and integrity of these sensors must be checked regularly 
to insure its accurate and dependable operation [6-10]. Additionally, if a vehicle manufacturer adapts low-cost sensors, the accuracy and reliability of these sensors must be regularly checked to insure its accurate functionality.

In this paper, a fault detection and identification routine is proposed to check the integrity of vehicle's ultrasonic sensors. The observations of the sensors are first manipulated to form an independent sequence in time that is only a function of the measurement noise and the possible measurement fault. A bank of possible sensors' faults, hypotheses, is constructed, and the probability of each hypothesis is calculated given the measurements of the sensors. The hypotheses are initialized with equal probability and subsequently updated in time until the correct hypothesis is identified. The identified hypothesis will have a probability that approaches one. The result of this study forms an essential component of a vehicle's total integrity assurance.

The outline of this paper is as follows. Section 2 gives an introduction to ultrasonic sensors' measurements and their failure modes. Section 3 gives an overview of fault detection as applied to ultrasonic sensors' measurements and introduces the probabilistic fault detection and identification algorithm proposed in this paper. The construction of the residual process that is a function of the measurement noise and the possible added sensor fault will be described. In Section 4, the platform used to check the algorithm is described and sensors' measurement model is introduced. The algorithm will be validated using two vehicle-barrier orientations. In Section 5, the method is validated through various experimental tests. Section 6 finally concludes the findings of this study.

\section{Physical Background on Ultrasonic Sensors and Their Failure Modes}

Ultrasonic sensors' measurements are based on transmitting ultrasonic waves and subsequently receiving the wave after it is reflected back from the object. By measuring the wave's time-of-flight (TOF), the range to the object is obtained.

The reflected echo of the ultrasonic sensor's sound wave has a time envelope that is characterized by the following equation $[6-8]$ :

$$
A(t)=A_{0}\left(\frac{t-T}{T}\right)^{\alpha} \exp \left(\frac{t-\tau}{T}\right),
$$

where $A(t)$ is the received signal value at time $t, A_{0}$ is the echo amplitude, $\tau$ is the TOF of the signal, and $\alpha$ and $T$ are distinct to the specific ultrasonic transducer used. To determine the correct range from the sensor to the obstacle, or object, in front of the sensor, the precise location of the received echo onset must be determined. If the echo starting time is not determined accurately due to the environment of the sensor, the object orientation, or object surface properties, then the sensor's range measurement will be biased [6].

The ultrasonic sensors are usually equipped with a temperature sensor in order to compensate for the change in the speed of sound which depends on temperature. If the temperature sensor's measurement is biased, a resulting bias in the ultrasonic sensor's range to the object will occur [6-8].
Researchers have worked on determining whether one of the ultrasonic sensors in a sensor array is faulty by having an erroneous measurement that is not consistent with the measurements of the other sensors used in the application $[9,10]$.

Due to these reasons, this paper proposes a method for detecting and identifying a possible bias error in the ultrasonic sensors' measurements. In the next section, an overview of fault detection theory is given with emphasis on fault detection and identification of ultrasonic sensors' measurements.

\section{Fault Detection and Identification}

As mentioned in the previous section, ultrasonic sensors can acquire a bias in their measurement due to various reasons. Some researchers attempted to reduce the bias in the sensor's readings by estimating the parameters of the reflected sensor's echo [6-8]. Since the model of the echo envelope, as given in (1), is nonlinear, their method required linearizing the equation about nominal envelope parameters and subsequently using linearized estimation techniques to obtain updated estimates of the echo envelope parameters, of which the TOF is one. The estimation techniques used were the linearized least-square estimator or the discrete extended Kalman filter. The methods in these studies depend on processing the measurements of only one ultrasonic sensor. If the sensor acquires a large bias error, then a convergence problem is expected in their proposed estimation method [23]. In severe conditions, the linearized estimator might even diverge [23]. On the other hand, this paper proposes a nonlinear sequential method that depends on the simultaneous sampling and processing of the measurements of more than one sensor. Therefore, even if one of the sensors acquires a bias, then the output of the linearized estimator, used in the proposed probabilistic sequential method, will not be much affected.

In $[9,10]$, authors proposed a method for detecting a failure in some of the ultrasonic sensors used in autonomous mobile robots. The used autonomous robot is equipped with 24 ultrasonic sensors. In their study, a faulty sensor was probabilistically detected by identifying inconsistencies among sensors' readings. While their study detected the faulty sensor, it did not identify the error in that sensor. In comparison, the proposed method in this paper detects the faulty sensor and identifies the bias error that is incorporated in its measurement. This allows to continue using the sensor by subtracting the identified bias from its measurements. Also, in the current method, only four sensors are needed to detect and identify a possible bias error in the measurement.

Estimating the mean of a random variable with known covariance from a set of two possible hypotheses was first treated through a sequential hypothesis testing methodology in [22]. Independent and identically distributed measurements, $r_{k}$, driven by white Gaussian noise are sampled and from which a statistical test is used to detect the correct hypothesis. The test is based on the ratio of the probability of having hypothesis 1 as the correct hypothesis, $p_{1}(k)$, to probability of hypothesis 0 as the correct hypothesis, $p_{0}(k)$, 
given all the measurements up to time $k$. This ratio can be shown to be

$$
\frac{p\left(H_{1} \mid r_{1}, r_{2}, \ldots, r_{k}\right)}{p\left(H_{0} \mid r_{1}, r_{2}, \ldots, r_{k}\right)}=\frac{p\left(r_{1} \mid H_{1}\right) p\left(r_{2} \mid H_{1}\right) \cdots p\left(r_{k} \mid H_{1}\right)}{p\left(r_{1} \mid H_{0}\right) p\left(r_{2} \mid H_{0}\right) \cdots p\left(r_{k} \mid H_{0}\right)} \text {. }
$$

Hypothesis 0 is accepted if this ratio is less than a specified lower threshold. Alternatively, hypothesis 1 is accepted if the above ratio exceeds another specified upper threshold. The used lower and upper thresholds are based on a selected bound for the false alarm probability of declaring hypothesis 0 as the correct hypothesis when hypothesis 1 is the true hypothesis and on the alternative false alarm bound of declaring hypothesis 1 as the correct hypothesis when hypothesis 0 is the true hypothesis.

In the problem addressed in this paper, each sensor can acquire one of five possible bias hypotheses. The bias hypotheses that are considered are a zero bias hypothesis, $-10 \mathrm{~cm}$ bias hypothesis, $-5 \mathrm{~cm}$ bias hypothesis, $5 \mathrm{~cm}$ bias hypothesis, and a $10 \mathrm{~cm}$ bias hypothesis. In addition, the vehicle is equipped with multiple sensors with each possibly incurring a bias. This fact enlarges the hypothesis set even more. Therefore, the proposed approach is based on monitoring the probability of having each hypothesis as the measurements are sampled. This approach is described as follows.

Given a residual $r_{k}$ at time $k$, with possible fault $H_{i}$, it can be shown that the probability of having the fault $H_{i}$ given residuals $r_{1}$ through $r_{k+1}$ is given by [22]

$$
p\left(H_{i} \mid r_{1}, r_{1}, \ldots, r_{k+1}\right)=\frac{p\left(H_{i}, r_{k+1} \mid r_{1}, r_{1}, \ldots, r_{k}\right)}{p\left(r_{k+1} \mid r_{1}, r_{1}, \ldots, r_{k}\right)},
$$

where the fault hypothesis $H_{i}$ is the event of having a bias $\mu_{i}$, $\mu_{i} \sim N\left(\bar{\mu}_{i}, \sigma_{M}^{2}\right)$, in one of the ultrasonic sensors' measurements. Defining $F_{i}(k)=p\left(H_{i} \mid r_{1}, r_{2}, \ldots, r_{k}\right)$, the following is obtained [22]:

$$
\begin{aligned}
F_{i}(k+1) & =\frac{p\left(r_{k+1} \mid H_{i}, r_{1}, r_{2}, \ldots, r_{k}\right) p\left(H_{i} \mid r_{1}, r_{2}, \ldots, r_{k}\right)}{p\left(r_{k+1} \mid r_{1}, r_{2}, \ldots, r_{k}\right)} \\
& =\frac{p\left(r_{k+1} \mid H_{i}\right) F_{i}(k)}{\sum_{i=1}^{m} p\left(r_{k+1}, H_{i} \mid r_{1}, r_{2}, \ldots, r_{k}\right)} \\
& =\frac{p\left(r_{k+1} \mid H_{i}\right) F_{i}(k)}{\sum_{i=1}^{m} p\left(r_{k+1} \mid H_{i}\right) p\left(H_{i} \mid r_{1}, r_{2}, \ldots, r_{k}\right)}
\end{aligned}
$$

or:

$$
F_{i}(k+1)=\frac{p\left(r_{k+1} \mid H_{i}\right) F_{i}(k)}{\sum_{i=1}^{m} p\left(r_{k+1} \mid H_{i}\right) F_{i}(k)} .
$$

The residual used in (5) is now formulated and from which the various probability terms that appear in the equation will be obtained. If the range between the ultrasonic sensor $i$ and the obstacle in front of it is given by $\Delta x_{i}$, then its associated measured range is given by

$$
\rho_{i}=\Delta x_{i}+v+\mu_{i},
$$

where $v$ is the sensor's measurement noise that is assumed normally distributed with zero mean and covariance $\sigma^{2}$, that is, $v \sim N\left(0, \sigma^{2}\right)$, and $\mu_{i}$ is the fault in sensor $i$ as defined below (3). If a sensor has a nonzero measurement noise mean, then this noise mean can be compensated for as an added bias fault.

The measurements from all ultrasonic sensors can be put in a vector equation as

$$
y=H \Delta x+v+\mu,
$$

where $y$ is the measurement vector composed from all sensors measurements, $v$ is the measurement noise vector composed from all sensors' measurement noise terms, and $\mu$ is the measurements fault vector composed from all sensors' measurement fault terms. The aim now is to form a residual that is a function of the measurement fault only and is independent of the distance between the sensor and the obstacle. To obtain this residual, an estimate of $\Delta x$ is first obtained as [23]

$$
\Delta \widehat{x}=\left(H^{T} V^{-1} H\right)^{-1} H^{T} V^{-1} y,
$$

where $V$ is the covariance matrix of the measurement noise vector $v$. The sensors' measurement noise terms are assumed independent and identically distributed (iid). Therefore, $V$ is taken as a diagonal matrix with a value of $\sigma^{2}$ on the diagonal. Then, the residual is formed as [21]

$$
\begin{aligned}
r & =y-H \Delta \hat{x} \\
& =y-H\left(H^{T} V^{-1} H\right)^{-1} H^{T} V^{-1} y \\
& =P y \\
& =P(H \Delta x+v+\mu) \\
& =P(v+\mu),
\end{aligned}
$$

where the annihilator $P=I-H\left(H^{T} H\right)^{-1} H^{T}$ makes the residual independent of $\Delta x$. In forming this annihilator, the fact that the measurement noise terms are iid was utilized.

Now, since the annihilator $P$ is rank deficient, its inverse is not defined. Therefore, performing a singular value decomposition of $P$ gives

$$
\begin{aligned}
P & =\left[\begin{array}{ll}
U_{1} & U_{2}
\end{array}\right]\left[\begin{array}{ll}
I & 0 \\
0 & 0
\end{array}\right]\left[\begin{array}{l}
V_{1} \\
V_{2}
\end{array}\right] \\
& =U_{1} V_{1},
\end{aligned}
$$

where $U_{1}^{T} U_{1}=I$. Therefore, the residual can be written as

$$
r=U_{1} V_{1}(v+\mu)
$$

Premultiplying the above equation by $U_{1}^{T}$, the following modified residual is obtained:

$$
\tilde{r}=U_{1}^{T} r=V_{1}(v+\mu),
$$


where $\tilde{r}$ is a residual that is independent of $\Delta x$ with the following conditional mean:

$$
\bar{r}=E\left[\tilde{r} \mid H_{i}\right]=V_{1} \bar{\mu}=V_{1}\left[\begin{array}{c}
0 \\
\bar{\mu}_{i} \\
0 \\
0
\end{array}\right],
$$

where it will be assumed that only one sensor will have a fault at any given time. Defining

$$
\Delta \widetilde{r}\left|H_{i}=(\tilde{r}-\bar{r})\right| H_{i}=V_{1} v-V_{1}\left[\begin{array}{c}
0 \\
\mu_{i}-\bar{\mu}_{i} \\
0 \\
0
\end{array}\right],
$$

the residual covariance can be obtained as

$$
E\left[\Delta \tilde{r} \Delta \widetilde{r}^{T} \mid H_{i}\right]=\Lambda=\sigma^{2} V_{1} V_{1}^{T}+\sigma_{M}^{2} V_{1}^{i} V_{1}^{i T},
$$

where $V_{1}^{i}$ is the $i$ th column of $V_{1}$. Now, the probability mass function of having the residual $\widetilde{r}_{k}$ given hypothesis $H_{i}$ can be formulated as

$$
p\left(\widetilde{r}_{k+1} \mid H_{i}\right)=\frac{1}{(2 \pi)^{n / 2}|\Lambda|^{1 / 2}} e^{-(1 / 2)(\tilde{r}-\bar{r})^{T} \Lambda^{-1}(\tilde{r}-\bar{r})} .
$$

At each measurement epoch, the above expression will be evaluated and substituted in (5). All hypotheses will be initialized to be equally probable.

In the next section, the experimental setup used in this study will be described. Also, the model of the ultrasonic sensors that is used to calculate the probabilities of the various hypotheses using (5) will be shown.

\section{Experimental Setup and System Model}

Figure 1 shows the experimental setup used to validate the algorithm presented in this paper. Four ultrasonic sensors were fixed uniformly along the front of a robot. The sensors used are the Devantech SRF04 ultrasonic sensors. Table 1 lists the specifications of these ultrasonic sensors.

To verify the integrity of the FDI algorithm, the robot was initially aligned to face a wall as shown in Figure 1. In this alignment, all four ultrasonic sensors are measuring the same range $\Delta x$.

The measurements of the four sensors can be put in equation form as

$$
\left[\begin{array}{l}
\rho_{1} \\
\rho_{2} \\
\rho_{3} \\
\rho_{4}
\end{array}\right]=\left[\begin{array}{l}
1 \\
1 \\
1 \\
1
\end{array}\right] \Delta x+\left[\begin{array}{l}
v_{1} \\
v_{2} \\
v_{3} \\
v_{4}
\end{array}\right]+\left[\begin{array}{l}
\mu_{1} \\
\mu_{2} \\
\mu_{3} \\
\mu_{4}
\end{array}\right],
$$

or, in matrix form,

$$
y=H \Delta x+v+\mu,
$$

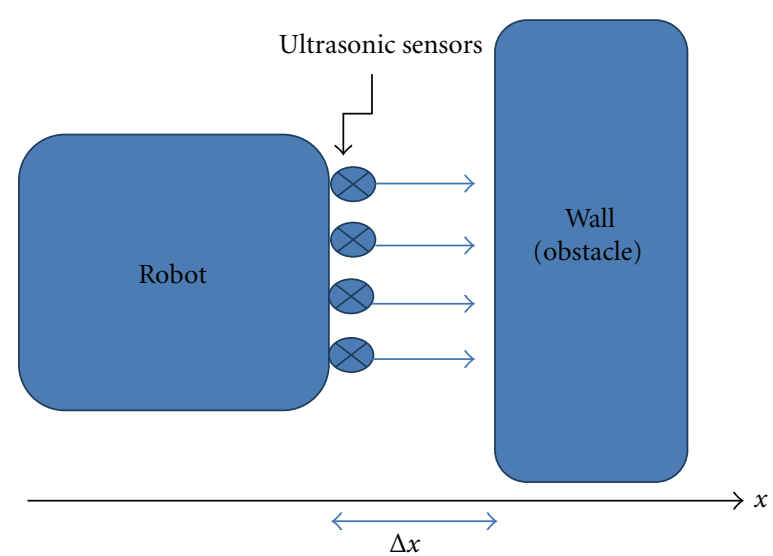

Figure 1: Experimental setup A.

TABle 1: Specifications of the ultrasonic sensors used.

\begin{tabular}{lc}
\hline Voltage & $5 \mathrm{v}$ \\
Current & $30 \mathrm{~mA}-50 \mathrm{~mA}$ \\
Frequency & $40 \mathrm{KHz}$ \\
Maximum range & $3 \mathrm{~m}$ \\
Minimum range & $3 \mathrm{~cm}$ \\
Sensitivity & Detect a $3 \mathrm{~cm}$ diameter stick at $>2 \mathrm{~m}$ \\
Weight & $0.4 \mathrm{oz}$. \\
Size & $1.75^{\prime \prime} \mathrm{w} \times 0.625^{\prime \prime} \mathrm{h} \times 0.5^{\prime \prime} \mathrm{d}$
\end{tabular}

where, as shown in the above equation, $y$ is the vector of sensors' measurements, $H$ is a vector of ones, $v$ is the vector of sensors' measurements' noise, and $\mu$ is the vector of sensors' measurements bias.

Measurements from the sensors were recorded. A fault was simulated on one of the sensors, and the presented FDI method was used to detect the faulty sensor and the magnitude of the fault as will be shown in the next section.

Subsequently, the robot was aligned at an angle from the wall as shown in Figure 2. In this alignment, each sensor is measuring a different range to the wall. Nevertheless, all measurements are a function of the orientation of the robot relative to the wall. The measurements from the first two sensors are given as

$$
\begin{aligned}
& \rho_{1}=\Delta x_{1}+v_{1}, \\
& \rho_{2}=\Delta x_{2}+v_{2} .
\end{aligned}
$$

But $\Delta x_{1}$ and $\Delta x_{2}$ are related by

$$
\frac{\Delta x_{1}}{a}=\frac{\Delta x_{2}}{a+d},
$$

where $a$ is the distance from the first ultrasonic sensor to the wall and $d$ is the separation distance between the sensors, as shown in Figure 2.

Similarly, it is easily shown that

$$
\frac{\Delta x_{1}}{a}=\frac{\Delta x_{3}}{a+2 d}=\frac{\Delta x_{4}}{a+3 d} .
$$




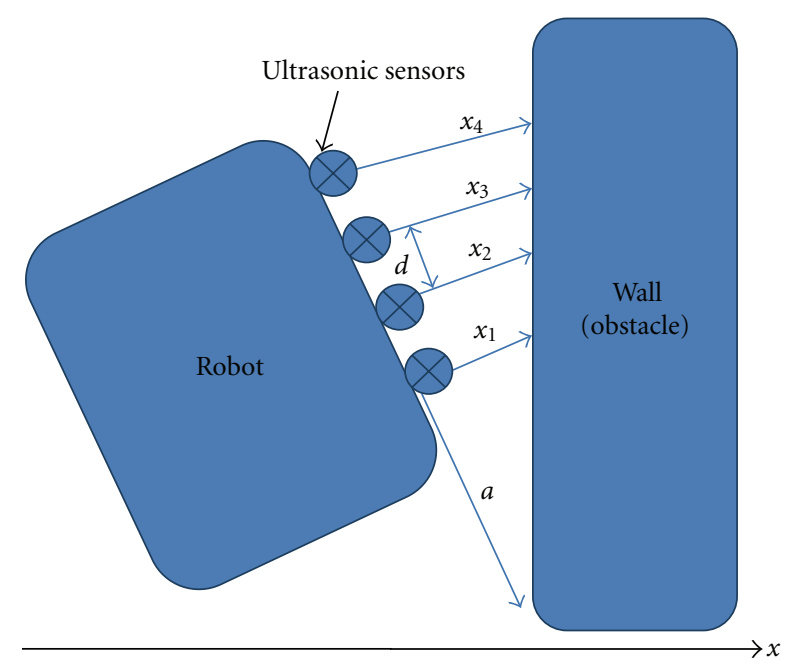

Figure 2: Experimental setup B.

Therefore, the measurements of the sensors are formulated to be a nonlinear function of the distance $\Delta x_{1}$ and the distance $a$ as:

$$
\begin{aligned}
& \rho_{1}=\Delta x_{1}+v_{1}+\mu_{1}, \\
& \rho_{2}=\frac{a+d}{a} \Delta x_{1}+v_{2}+\mu_{2}, \\
& \rho_{3}=\frac{a+2 d}{a} \Delta x_{1}+v_{3}+\mu_{3}, \\
& \rho_{4}=\frac{a+3 d}{a} \Delta x_{1}+v_{4}+\mu_{4} .
\end{aligned}
$$

These measurements are linearized about nominal $\Delta x_{1}$ and $a$ values to obtain

$$
\rho_{i}=\bar{\rho}_{i}+\left[\begin{array}{ll}
\frac{\partial \rho_{i}}{\partial \Delta x_{1}} & \frac{\partial \rho_{i}}{\partial a}
\end{array}\right]\left[\begin{array}{c}
\Delta \tilde{x} \\
\tilde{a}
\end{array}\right]+v_{i}+\mu_{i},
$$

where $\Delta \tilde{x}=\Delta x_{1}-\Delta \bar{x}_{1}, \tilde{a}=a-\bar{a}$, and $\bar{\rho}_{i}$ is the evaluated range of sensor $i$ obtained using the nominal values of $\Delta x=\Delta \bar{x}$ and $a=\bar{a}$. Similarly, the partial derivatives in (23) are evaluated using the nominal values. Therefore, the measurement equation can be written as:

$$
y=H \Delta x+v+\mu
$$

where

$$
H=\left[\begin{array}{cc}
0 & 1 \\
\frac{\bar{a}+d}{\bar{a}} & \frac{-d \Delta \bar{x}_{1}}{\bar{a}^{2}} \\
\frac{\bar{a}+2 d}{\bar{a}} & \frac{-2 d \Delta \bar{x}_{1}}{\bar{a}^{2}} \\
\frac{\bar{a}+3 d}{\bar{a}} & \frac{-3 d \Delta \bar{x}_{1}}{\bar{a}^{2}}
\end{array}\right],
$$

$y=\rho-\bar{\rho}, \Delta x=\left[\begin{array}{c}\Delta \tilde{x} \\ \tilde{a}\end{array}\right]$, with $v$ and $\mu$ are as defined before. Next, the fault hypotheses are defined and the experimental results are shown to validate the performance of the presented algorithm.

\section{Experimental Results}

To test the performance of the presented algorithm, data was collected from the four ultrasonic sensors with the two robot configurations shown in Figures 1 and 2. Faults of $10 \mathrm{~cm}$, $5 \mathrm{~cm}, 0 \mathrm{~cm},-5 \mathrm{~cm}$, and $-10 \mathrm{~cm}$ were hypothesized on each sensor. It was assumed that only one sensor will have a fault at any given time. Therefore, a total of 17 hypotheses need to be checked at each time. The hypotheses are defined as

$$
\begin{gathered}
H_{1}=\left[\begin{array}{l}
0 \\
0 \\
0 \\
0
\end{array}\right], \quad H_{2}=\left[\begin{array}{l}
10 \\
0 \\
0 \\
0
\end{array}\right], \quad H_{3}=\left[\begin{array}{l}
5 \\
0 \\
0 \\
0
\end{array}\right], \\
H_{4}=\left[\begin{array}{c}
-10 \\
0 \\
0 \\
0
\end{array}\right], \quad H_{5}=\left[\begin{array}{c}
-5 \\
0 \\
0 \\
0
\end{array}\right], \\
H_{6}=\left[\begin{array}{c}
0 \\
10 \\
0 \\
0
\end{array}\right], \ldots, H_{17}=\left[\begin{array}{c}
0 \\
0 \\
0 \\
-5
\end{array}\right] .
\end{gathered}
$$

Assuming that one sensor will fail at a time is logical since the method is intended to be applied periodically at the start of the vehicle operation once the vehicle is facing a barrier similar to that shown in Figure 1 or 2 . Also, the method can be applied in periodic maintenance procedures scheduled for the vehicle. Therefore, once a sensor is detected to have a bias, then that bias can be deducted from the sensor's measurement and the sensor set is reset to have a null bias state. Nevertheless, the method can incorporate more than one faulty sensor at a time if the number of the sensors would be increased, as an example from 4 to 5 , to keep the same amount of redundancy between the sensors. A problem would arise if, in 4 sensors, two acquire the same amount of bias at the same time. In this scenario, it is impossible for the method to decide on the healthy sensors. This is the reason why you need an additional sensor to detect the onset of two failed sensors at a time.

The algorithm starts with setting all hypotheses to be equally probable. As data is sampled, the probability of each hypothesis is sequentially updated. Once the probability associated with a hypothesis reaches a threshold close to 1, the fault associated with that hypothesis is declared. Below, experimental results showing the performance of the proposed algorithm are presented.

Figures 3 through 8 show the performance of the FDI algorithm tested when the robot is in the setup shown in Figure 1. The sensors were sampled in this position and subsequently a possible fault in any of the sensors was detected using the presented algorithm. Initially, no fault was added to the collected data. Therefore, the probability associated with hypothesis $H_{1}$, the null hypothesis, should converge 


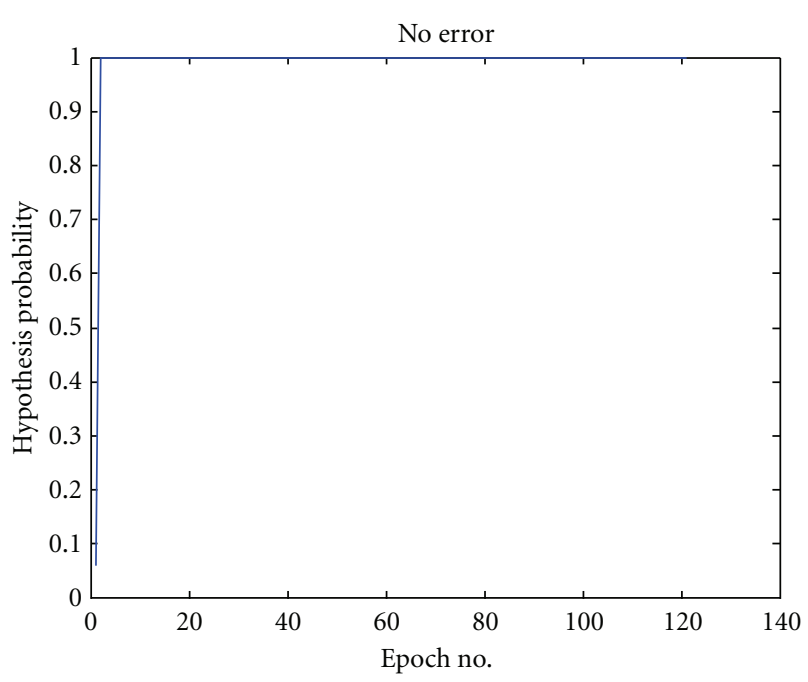

FIgURE 3: Probability associated with no fault hypothesis, setup A.

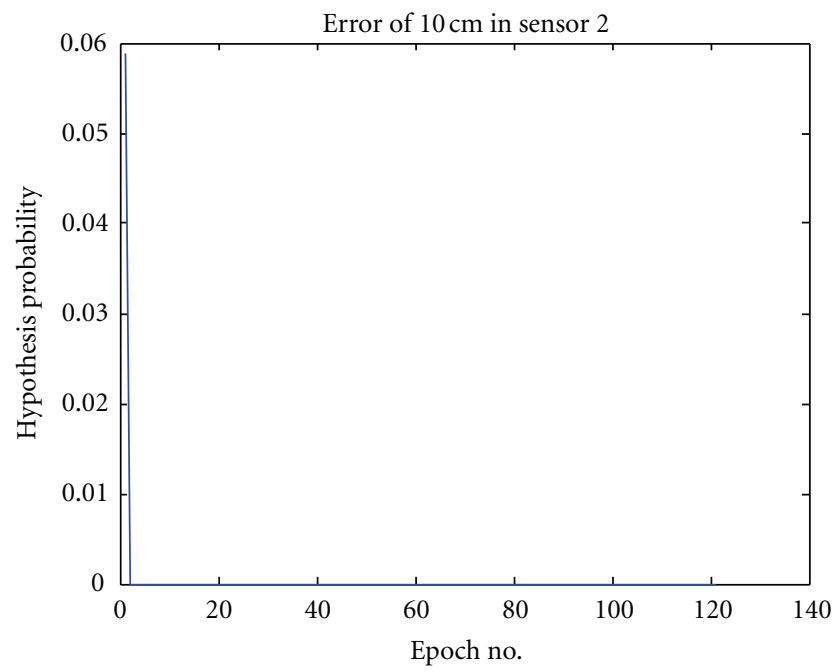

Figure 4: Probability associated with $10 \mathrm{~cm}$ fault on sensor 2, setup A.

to 1 . Figures 3 to 5 show the probability associated with some hypotheses in the hypothesis set. It can be seen from Figure 3 that the probability associated hypothesis $H_{1}$ quickly approaches 1 . Figures 4 and 5 show that the probability associated with hypothesis $H_{6}$, a bias of $10 \mathrm{~cm}$ on sensor 2, and that of hypothesis $H_{13},-5 \mathrm{~cm}$ on sensor 3, respectively, both quickly converge to 0 . These results validate the performance of the algorithm in this condition.

Figures 6 through 8 show the performance of the algorithm when a bias of $-10 \mathrm{~cm}$ was added to the measurements of sensor 4 . It can be seen that the probability associated with hypothesis $H_{1}$, the null hypothesis, and that of hypothesis $\mathrm{H}_{2}$, a fault of $10 \mathrm{~cm}$ on sensor 1 , quickly converge to zero as shown in Figures 6 and 7, respectively. Figure 8 shows that the probability associated with hypothesis $H_{16}$, the correct hypothesis, converges to 1 .

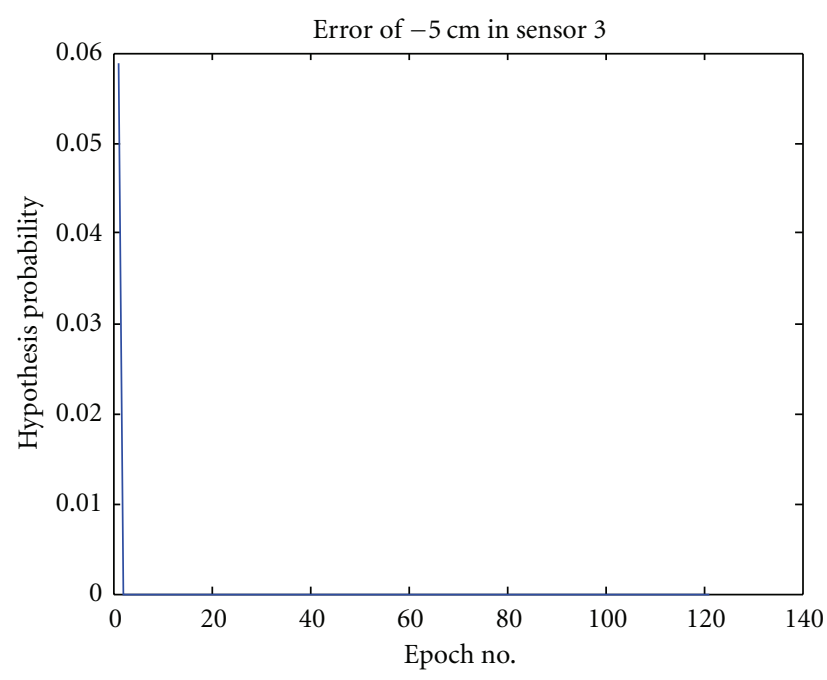

FIGURE 5: Probability associated with $-5 \mathrm{~cm}$ fault on sensor 3 , setup A.

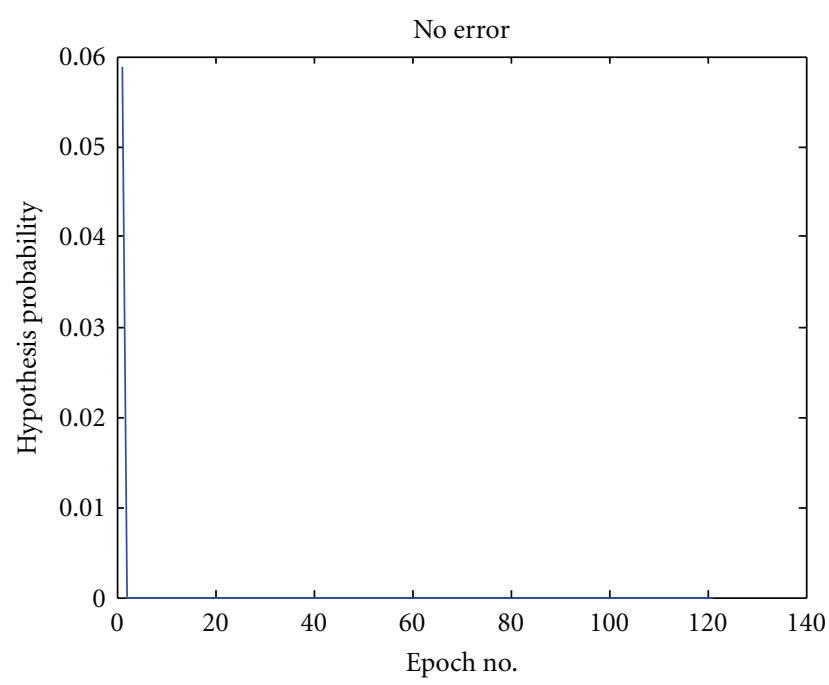

FIgURE 6: Probability associated with no fault hypothesis, setup A.

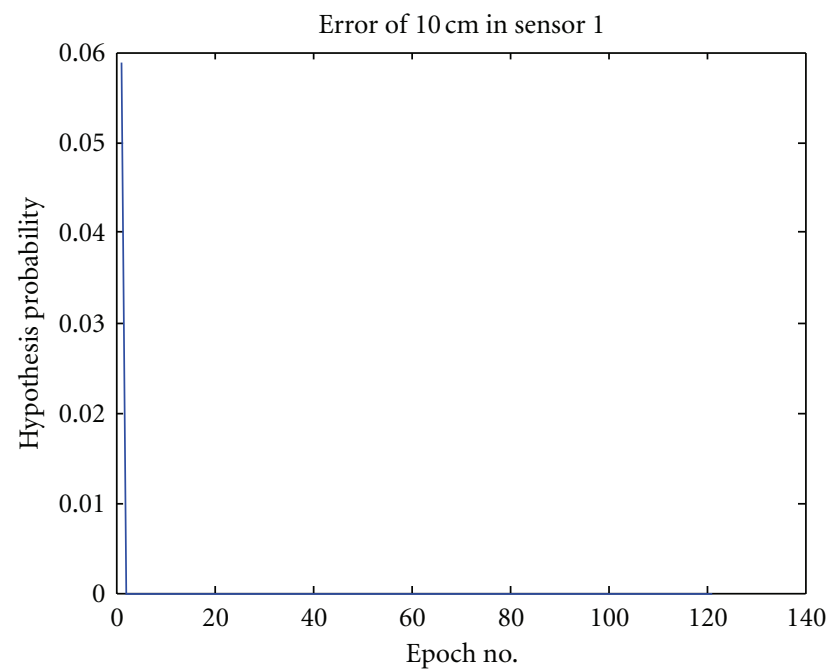

FIgure 7: Probability associated $10 \mathrm{~cm}$ bias on sensor 1, setup A. 


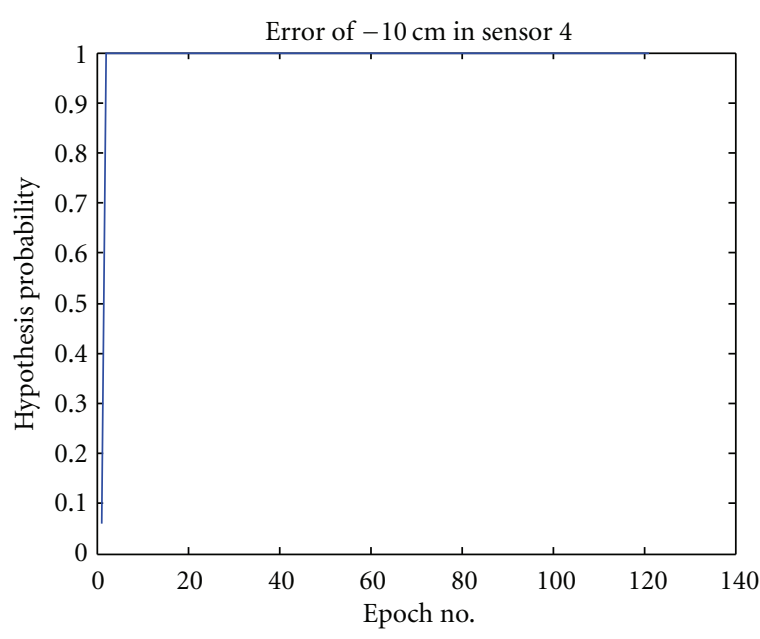

Figure 8: Probability associated $-10 \mathrm{~cm}$ bias on sensor 4 , setup A.

Figures 9 through 13 show the performance of the algorithm when the robot and the wall are at an angle from each other as shown in Figure 3. First, Figures 9 through 11 show the performance of the algorithm when a bias of $-10 \mathrm{~cm}$ is added to sensor 3. Figures 9 and 11 show that the probability associated with hypothesis $H_{1}$, the null hypothesis, and that associated with hypothesis $H_{16}$, a bias of $-10 \mathrm{~cm}$ on sensor 4 , respectively, quickly converge to 0 . On the other hand, Figure 10 shows that the probability associated with hypothesis $H_{12}$, a bias of $-10 \mathrm{~cm}$ on the third sensor, approaches 1.0 and therefore is declared the correct hypothesis.

Figures 12 and 13 show the performance of the method when a bias of $5 \mathrm{~cm}$ was added to the measurements of sensor 2. Figure 12 shows the probability associated with hypothesis $\mathrm{H}_{5}$, a bias of $-5 \mathrm{~cm}$ on sensor 1 , quickly converges to 0 probability. On the other hand, Figure 13 shows that the probability associated with hypothesis $H_{7}$, a bias of $5 \mathrm{~cm}$ on sensor 2, converges to a probability of 1.0 . This validates the accuracy of the presented algorithm in detecting a bias in any of the four ultrasonic sensors.

The performance of multihypothesis sequential probability ratio tests (MSPRTs) has been extensively studied in the literature, [24-26]. Nevertheless, exact expressions for the tests' expected stopping time and error probabilities are impossible to obtain except in special cases [24].

The stopping time $N_{A}$ of the MSPRT is defined as $N_{A}=$ first $n \geq 1$ such that $F_{i}(n)>1 /\left(1+A_{i}\right)$ for at least one $i$.

From (1), the stopping time can be written as $N_{A}=$ first $n \geq 1$ such that

$$
\frac{p\left(r_{n} \mid H_{i}\right) F_{i}(n-1)}{\sum_{i=1}^{m} p\left(r_{n} \mid H_{i}\right) p\left(H_{i} \mid r_{1}, r_{2}, \ldots, r_{n-1}\right)}>\frac{1}{1+A_{i}}
$$

for at least one $i$. The typical design values of $A_{i}$ is less than one. In this paper, $A_{i}$ is assumed constant for all values of $i$ with a value of 0.02 such that a hypothesis is accepted if its probability exceeds 0.98 .

Expressions for the bounds of the expected stopping time of the MSPRTs were obtained in reference [24]. Conditioned on the true hypothesis $k$, with the residual $r_{k}$ governed by a

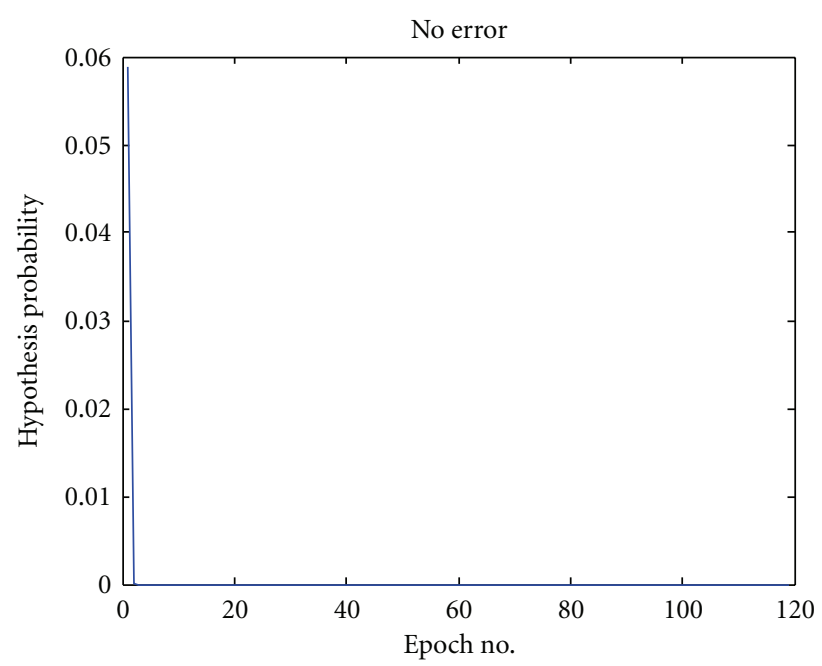

FIGURE 9: Probability associated with no fault hypothesis, setup B.

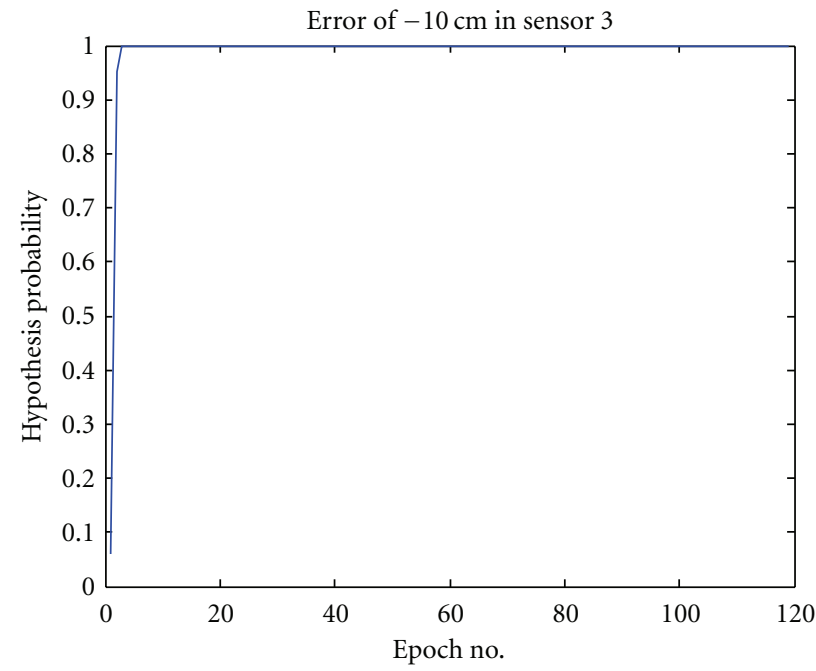

FIgure 10: Probability associated with $-10 \mathrm{~cm}$ bias on sensor 3, setup B.

probability density $f_{k}$, the expected stopping time approaches [24]

$$
\frac{E_{f_{k}}\left[N_{A}\right]}{-\log A_{k}} \longrightarrow \frac{1}{\min _{j: j \neq k} D\left(f_{k}, f_{j}\right)}
$$

as $\max _{l} A_{l} \rightarrow 0$, where the Kullback-Leibler distance function, $D(f, g)$, is given by

$$
D(f, g)=E_{f}\left[\log \frac{f(r)}{g(r)}\right]=\int f(r) \log \frac{f(r)}{g(r)} d r .
$$

In [24], the authors applied the equation above and obtained some numerical results for the first-order approximation of the stopping time of a three-hypothesis test. Higherorder approximations of the expected stopping time were obtained in references $[25,26]$ and applied for some two-hypothesis tests. Obtaining the expected value of the 


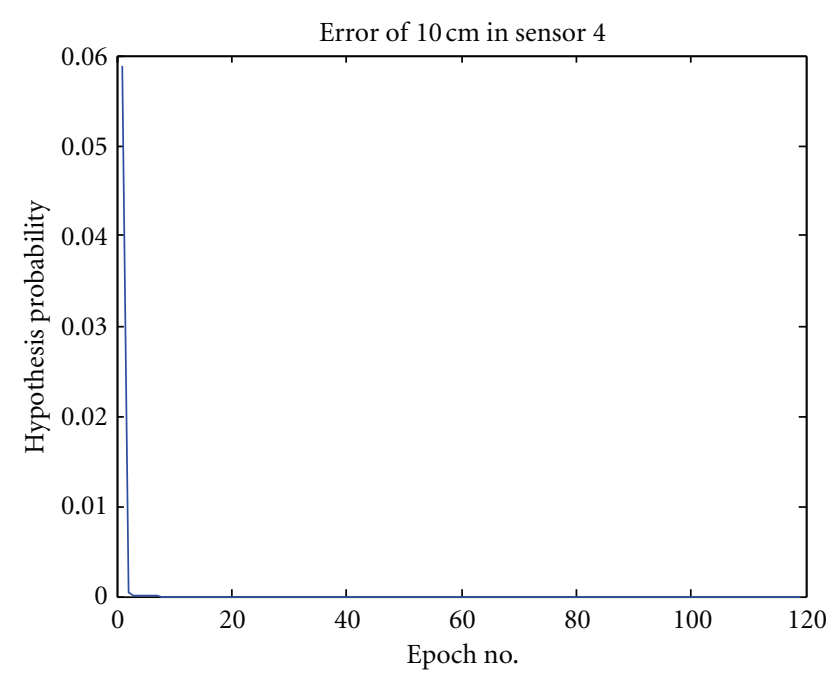

FIGURE 11: Probability associated with $10 \mathrm{~cm}$ bias on sensor 4, setup B.

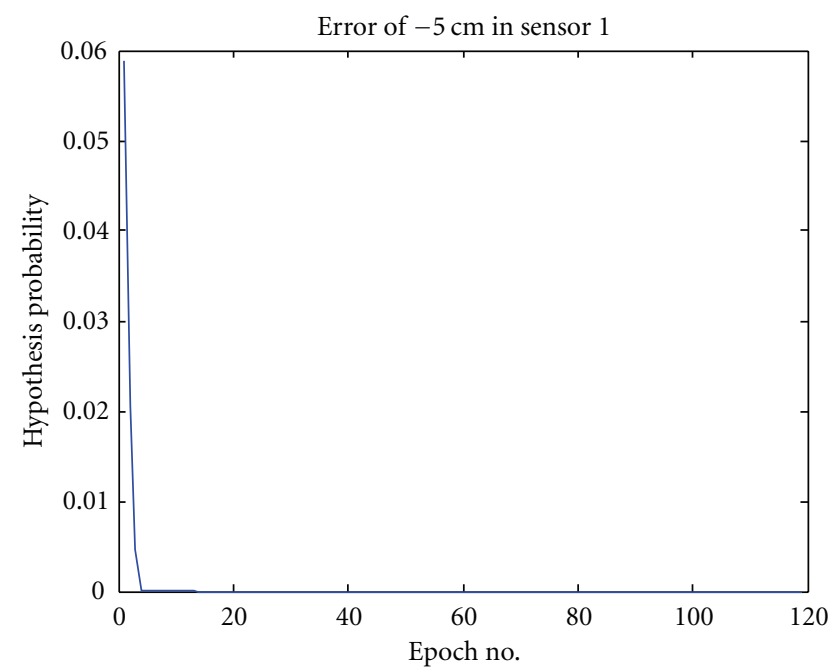

Figure 12: Probability associated with $-5 \mathrm{~cm}$ bias on sensor 1 , setup B.

stopping time for our multihypothesis problem, with 17 hypotheses, is very complicated and beyond the scope of this paper. However, the convergence characteristics of the problem at hand is analyzed numerically in Figures 14 and 15.

In Figure 14, the convergence characteristics of the method are examined by simulating varying measurement noise magnitudes and plotting the probability of the true hypothesis with time. In this test, setup B was used and the true hypothesis was $H_{3}, 5 \mathrm{~cm}$ bias on sensor 1 . It can be seen that, as the measurement noise is increased, the convergence to a probability of 1 takes more time.

Figure 15 shows a plot of the required measurement epochs for the algorithm's convergence as a function of the simulated measurement noise covariance. As in Figure 14, the results shown in this figure also belong to setup $B$ and the true hypothesis was $H_{3}, 5 \mathrm{~cm}$ bias on sensor 1 . The hypothesis was declared as the true hypothesis if its

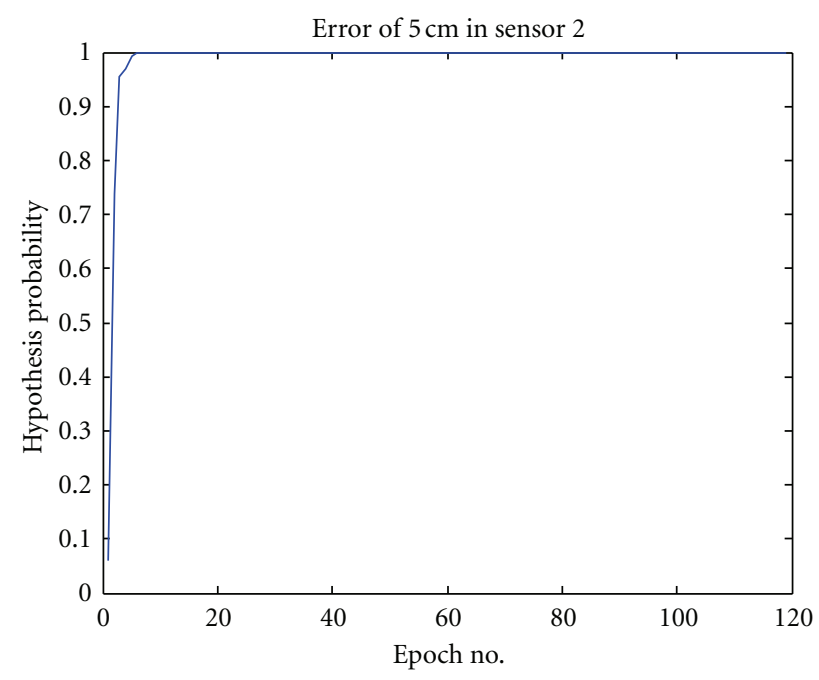

FIGURE 13: Probability associated with $5 \mathrm{~cm}$ bias on sensor 2, setup B.

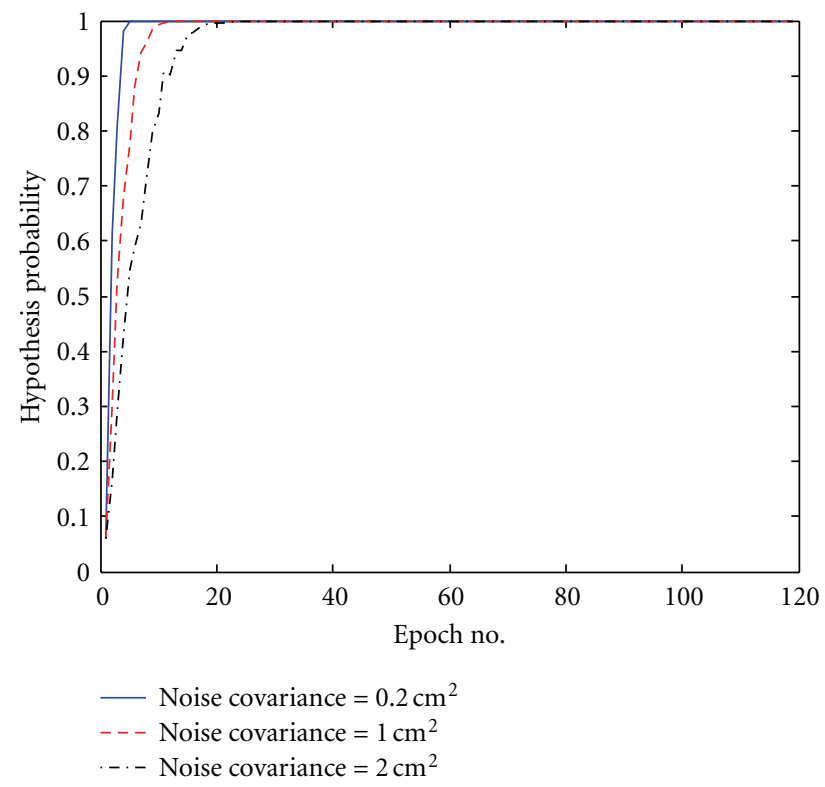

FIGURE 14: Convergence performance for different measurement noise magnitudes.

associated probability exceeded 0.98 . This choice of threshold is set by the user taking into account the convergence characteristics of the method. The choice for the convergence bounds is discussed in references $[19,20]$.

It can be shown that, in the mean, the probability of the true hypothesis will asymptotically converge to 1.0. This can be shown from the exponent of 11 evaluated at any given hypothesis $H_{i}$ :

$$
q_{i}(k)=-\frac{1}{2}\left(\tilde{r}(k)-\bar{r}_{i}\right)^{T} \Lambda^{-1}\left(\tilde{r}(k)-\bar{r}_{i}\right)
$$




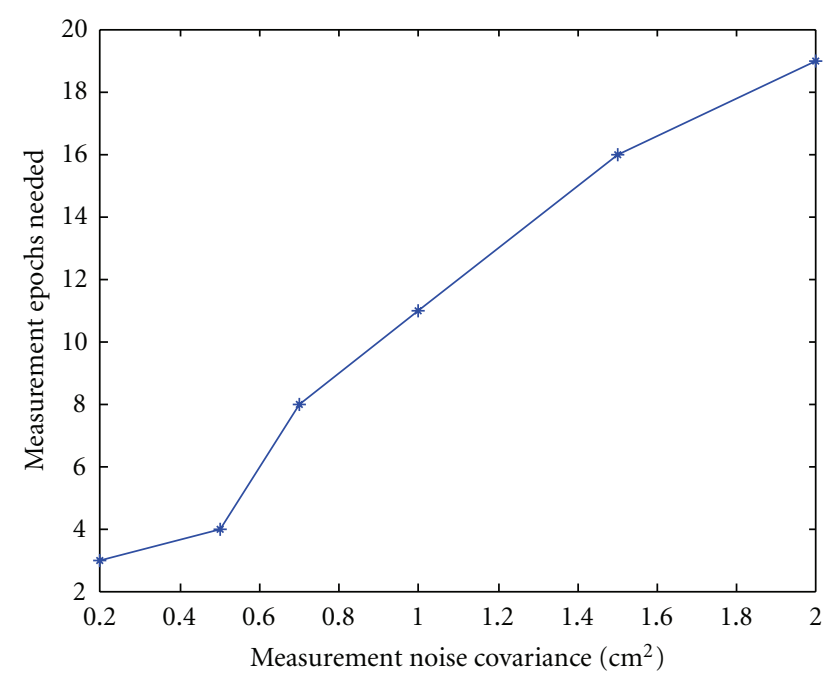

FIGURE 15: Number of epochs needed for algorithm's convergence for varying measurement noise covariance magnitude.

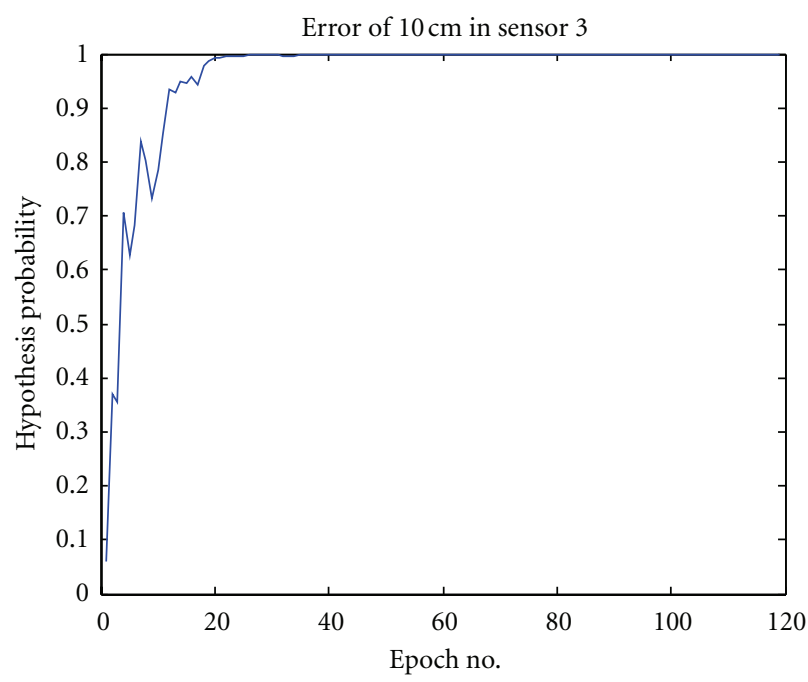

FIGURE 16: Probability associated with $10 \mathrm{~cm}$ bias on sensor 3, setup $\mathrm{B}$, true bias $=8 \mathrm{~cm}$.

At any given measurement epoch, the expected value of $q_{i}(k)$ is maximum if hypothesis $H_{i}$ is the true hypothesis $H_{t}$, that is:

$$
\max _{i=1}^{17} E\left[q_{i}(k)\right]=E\left[q_{i=t}(k)\right]
$$

where $E[x]$ denotes the expected value of the random process $x$. Therefore, in the mean, the probability mass function in (16) is maximum when evaluated for the true hypothesis $H_{t}$. This will, in the mean, make the probability of hypothesis $H_{t}$ asymptotically increase in time while the probability associated with all other hypotheses $H_{i}$ decrease in time as can be seen from (5).

If the true bias in one of the sensors exceeds the maximum bias hypothesis, then it can be seen from (31) that the algorithm will converge to the closest bias hypothesis.

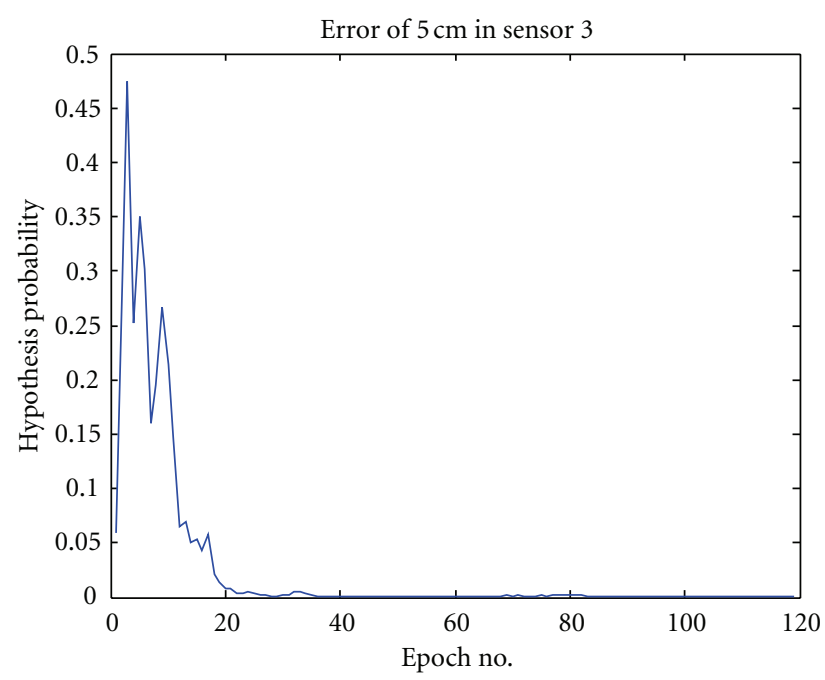

Figure 17: Probability associated with $5 \mathrm{~cm}$ bias on sensor 3 , setup $\mathrm{B}$, true bias $=8 \mathrm{~cm}$.

Subsequently, this bias will be deducted from the measurement of the sensor at each time and the hypotheses will then be redefined to check for the onset of another sensor's bias. Therefore, the method will continue to approach the real value of the bias in the sensors. Actually, regardless of which hypothesis the algorithm converged to, the fault hypotheses can be fine tuned around that hypothesis to obtain a more precise value of the true bias in the sensor. Such hypothesis redefinition and testing is feasible in view of the fast convergence characteristics of the algorithm and its small computational complexity as discussed below.

To compare the advantage of the proposed method against existing methods, a test was conducted using setup $\mathrm{B}$ where the true bias is simulated to be $8 \mathrm{~cm}$ on sensor 3. As listed at the beginning of this section, this bias is not one of the fault hypotheses tested in the algorithm. It rather falls between two hypotheses, namely, $H_{10}$ and $H_{11}$. Figures 16 and 17 show the performance of the algorithm in this test. The figures show that the algorithm initially gives about equal high probabilities to hypotheses $H_{10}$ and $H_{11}$ and finally converges to give a probability of about 1.0 to hypothesis $H_{10}$. This shows the accuracy and reliability of the proposed algorithm. The same test was repeated but with using only 3 sensors, instead of 4 as proposed by this algorithm, Figures 18 and 19. In this case, and as seen from the figure, the algorithm did not converge to any of the hypotheses close to the true hypotheses. The cause of this is believed to be attributed to the fact that the number of sensors' measurements is the same as the number of unknowns in the method, namely, $\Delta \tilde{x}, \tilde{a}$, and $\mu$, that is, there is no added redundancy. This last test simulates the problem that might be encountered in existing methods if the estimation algorithms used in the method is based on a linearized model.

The computational complexity of the method is small as the method is based on a least squares estimate of the range and the computation of the probability of each hypothesis through an exponential term, (16). Also, the singular value 


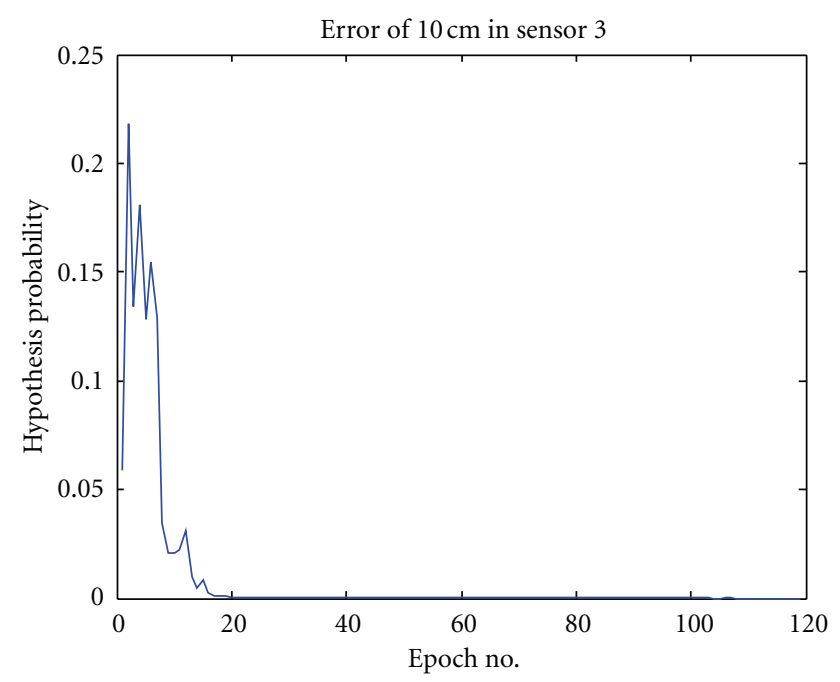

FIgure 18: Probability associated with $10 \mathrm{~cm}$ bias on sensor 3, setup $\mathrm{B}$, true bias $=8 \mathrm{~cm}$, only 3 sensors used.

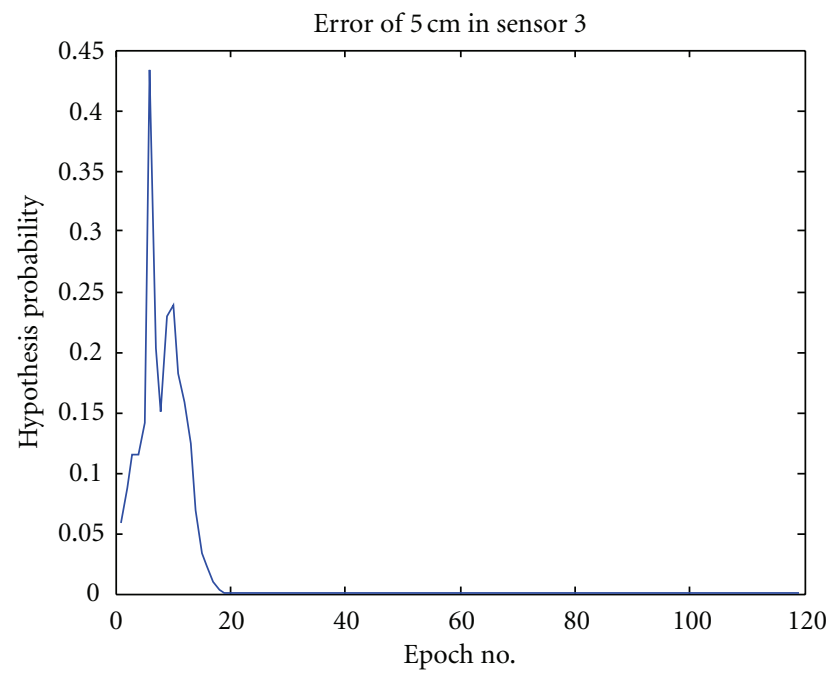

Figure 19: Probability associated with $5 \mathrm{~cm}$ bias on sensor 3, setup $\mathrm{B}$, true bias $=8 \mathrm{~cm}$, only 3 sensors used.

decomposition of a $4 \times 4$ matrix $P$ is not time consuming. The method was timed in MabLab on a Pentium IV platform, and it took less than 0.002 seconds for the processing of the measurements of the 4 sensors and obtaining the probability associated with all the hypotheses.

\section{Conclusions}

This study proposes a sequential fault detection and identification algorithm for vehicle's ultrasonic parking sensors. A number of fault hypotheses are initially given equal alarm probability. These probabilities are then updated in time as measurements from sensors are taken. When the probability of a certain hypothesis approaches 1 , the fault associated with that hypothesis is declared as the correct fault in the sensor set. Experimental results were shown to verify the accuracy of the algorithm in detecting and identifying the correct fault. The convergence characteristics of the algorithm were demonstrated. The proposed FDI algorithm is essential for ensuring the functionality and safety of operation of these sensors. The algorithm can be applied on vehicles' periodically scheduled maintenance routines.

\section{Acknowledgments}

The author wishes to thank Ahmed Al Nabulsi, Amir H. Jafari, Farouq Al Zaabi, Mohamad Sleiman, and Ahmad AbuHatab, class of the graduate mechatronics graduate course "Applied Linear Estimation," for collecting the experimental data used in this paper.

\section{References}

[1] I. V. Nikiforov, "New optimal approach to global positioning system/differential global positioning system integrity monitoring," Journal of Guidance, Control, and Dynamics, vol. 19, no. 5, pp. 1023-1033, 1996.

[2] B. W. Parkinson and P. Axelrad, "Autonomous GPS integrity monitoring using the pseudorange residual," Navigation, vol. 35, no. 2, pp. 255-274, 1988.

[3] K. Watanabe and D. M. Himmelbrau, "Instrument fault detection in systems with uncertainties," International Journal of System Science, vol. 13, pp. 483-500, 1982.

[4] M. F. Abdel-Hafez, "The autocovariance least-squares technique for GPS measurement noise estimation," IEEE Transactions on Vehicular Technology, vol. 59, no. 2, Article ID 5299067, pp. 574-588, 2010.

[5] N. A. White, P. S. Maybeck, and S. L. DeVilbiss, "Detection of interference/jamming and spoofing in a DCPS-aided inertial system," IEEE Transactions on Aerospace and Electronic Systems, vol. 34, no. 4, pp. 1208-1217, 1998.

[6] A. M. Sabatini, "A digital-signal-processing technique for ultrasonic signal modeling and classification," IEEE Transactions on Instrumentation and Measurement, vol. 50, no. 1, pp. 15-21, 2001.

[7] A. M. Sabatini, "Digital signal-processing technique for compensating ultrasonic sensors," IEEE Transactions on Instrumentation and Measurement, vol. 44, no. 4, pp. 869-874, 1995.

[8] L. Angrisani, A. Baccigalupi, and R. Schiano Lo Moriello, "A measurement method based on Kalman filtering for ultrasonic time-of-flight estimation," IEEE Transactions on Instrumentation and Measurement, vol. 55, no. 2, pp. 442-448, 2006.

[9] M. Soika, "Sensor failure detection framework for autonomous mobile robots," in Proceeding of the International Conference on Intelligent Robots and Systems, pp. 1735-1740, September 1998.

[10] D. Bank, "An error detection model for ultrasonic sensor evaluation on autonomous mobile systems," in Proceedings of the 11th IEEE International Workshop on Robot and Human Interactive, pp. 288-293, 2002.

[11] P. Frank, L. Palkovics, and L. Gianone, "Using wheel speed and wheel slip information for controlling vehicle chassis systems," in Proceedings of the 5th International Symposium on Advanced Vehicle Control (AVEC '00), 2000.

[12] S. L. Miller, B. Youngberg, A. Millie, P. Schweizer, and C. Gerdes, "Calculating longitudinal wheel slip and tire parameters using GPS velocity," in Proceedings of the American Controls Conference, pp. 1800-1805, June 2001. 
[13] F. J. J. Hermans and M. B. Zarrop, "Model based statistical change detection for automotive applications," in Proceedings of the IEEE International Symposium on Computer-Aided Control System Design, pp. 105-110, September 1996.

[14] M. L. Schwall and J. C. Gerdas, "Multi-modal diagnostics for vehicle fault detection," in Proceedings of the ASME International Mechanical Engineering Congress and Exposition, pp. 1753-1762, November 2001.

[15] M. L. Schwall and J. C. Gerdes, "A probabilistic approach to residual processing for vehicle fault detection," in Proceedings of the American Control Conference, pp. 2552-2557, May 2002.

[16] H. E. Tseng and L. Xu, "Robust model-based fault detection for roll rate sensor," in Proceedings of the 42nd IEEE Conference on Decision and Control, pp. 1968-1973, December 2003.

[17] O. Buehler and J. Wegener, "Evolutionary functional testing of an automated parking system," in Proceedings of the International Conference on Computer, Communication and Control Technologies and the 9th International Conference on Information Systems Analysis and Synthesis, pp. 1-8, 2003.

[18] N. Messai, P. Thomas, D. Lefebvre, and A. E. Moudni, "Neural networks for local monitoring of traffic magnetic sensors," Control Engineering Practice, vol. 13, no. 1, pp. 67-80, 2005.

[19] Shirayayev, Optimal Stopping Rules, Springer, New York, NY, USA, 1978.

[20] T. L. Lai, "Sequential multiple hypothesis testing and efficient fault detection-isolation in stochastic systems," IEEE Transactions on Information Theory, vol. 46, no. 2, pp. 595-608, 2000.

[21] S. Chan and J. L. Speyer, "A sequential probability test for RAIM," in Proceedings of the ION GNSS International Technical Meeting of the Satellite Division, pp. 1798-1802, September 2004.

[22] A. Wald, Sequential Analysis, Wiley Mathematical Statistics Series, Wiley Mathematical Statistics SeriesJohn Wiley and Sons, New York, NY, USA, 1947.

[23] Y. Bar-Shalom, X. R. Li, and T. Kirubarajan, Estimation with Applications to Tracking and Navigation, Theory, Algorithms, and Software, John Wiley \& Sons, New York, NY, USA, 2001.

[24] C. W. Baum and V. V. Veeravalli, "Sequential procedure for multihypothesis testing," IEEE Transactions on Information Theory, vol. 40, no. 6, pp. 1994-2007, 1994.

[25] V. P. Dragalin, A. G. Tartakovsky, and V. V. Veeravalli, "Multihypothesis sequential probability ratio tests-part I: asymptotic optimality," IEEE Transactions on Information Theory, vol. 45, no. 7, pp. 2448-2461, 1999.

[26] V. P. Dragalin, A. G. Tartakovsky, and V. V. Veeravalli, "Multihypothesis sequential probability ratio tests-part II: accurate asymptotic expansions for the expected sample size," IEEE Transactions on Information Theory, vol. 46, no. 4, pp. 1366-1383, 2000. 

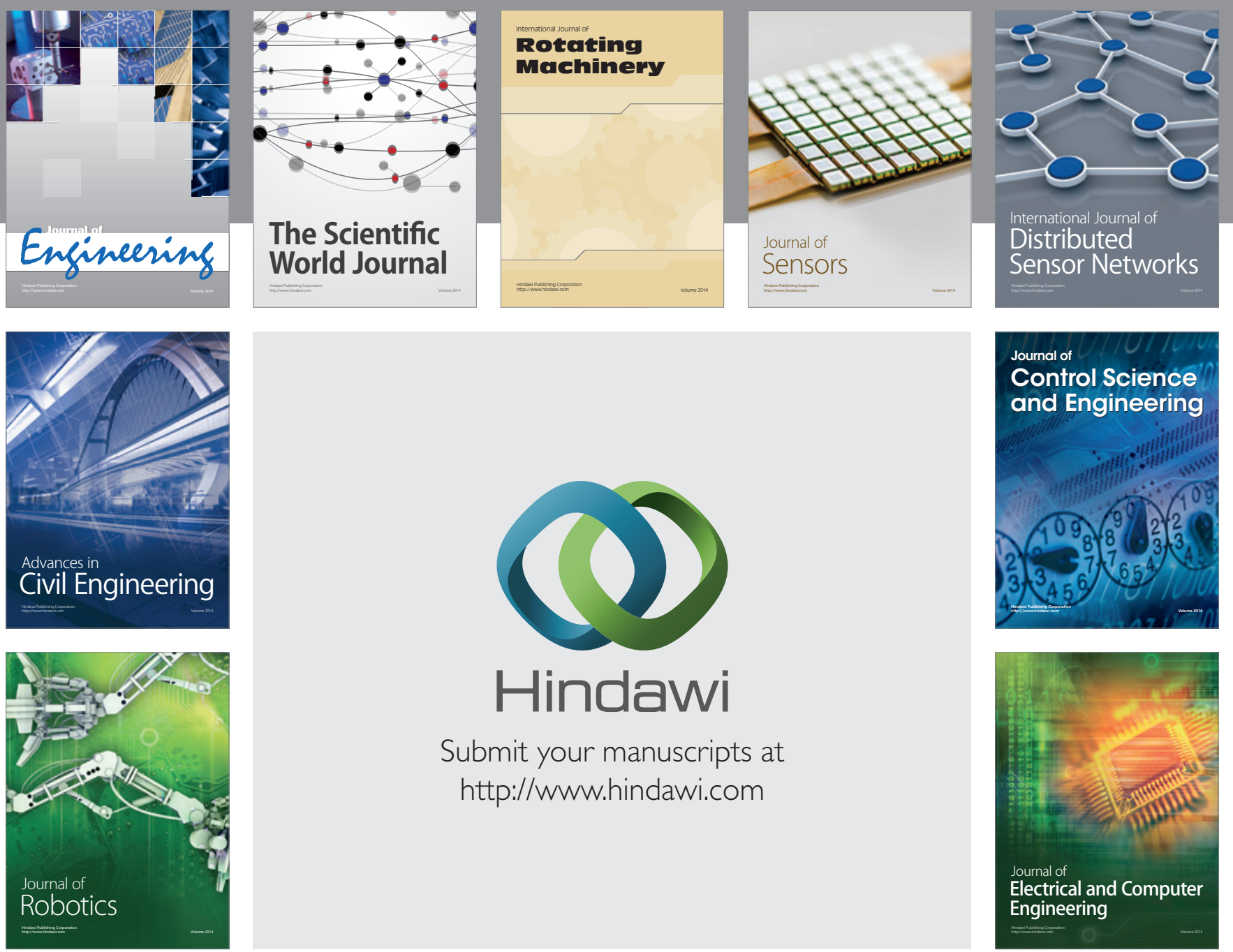

Submit your manuscripts at

http://www.hindawi.com
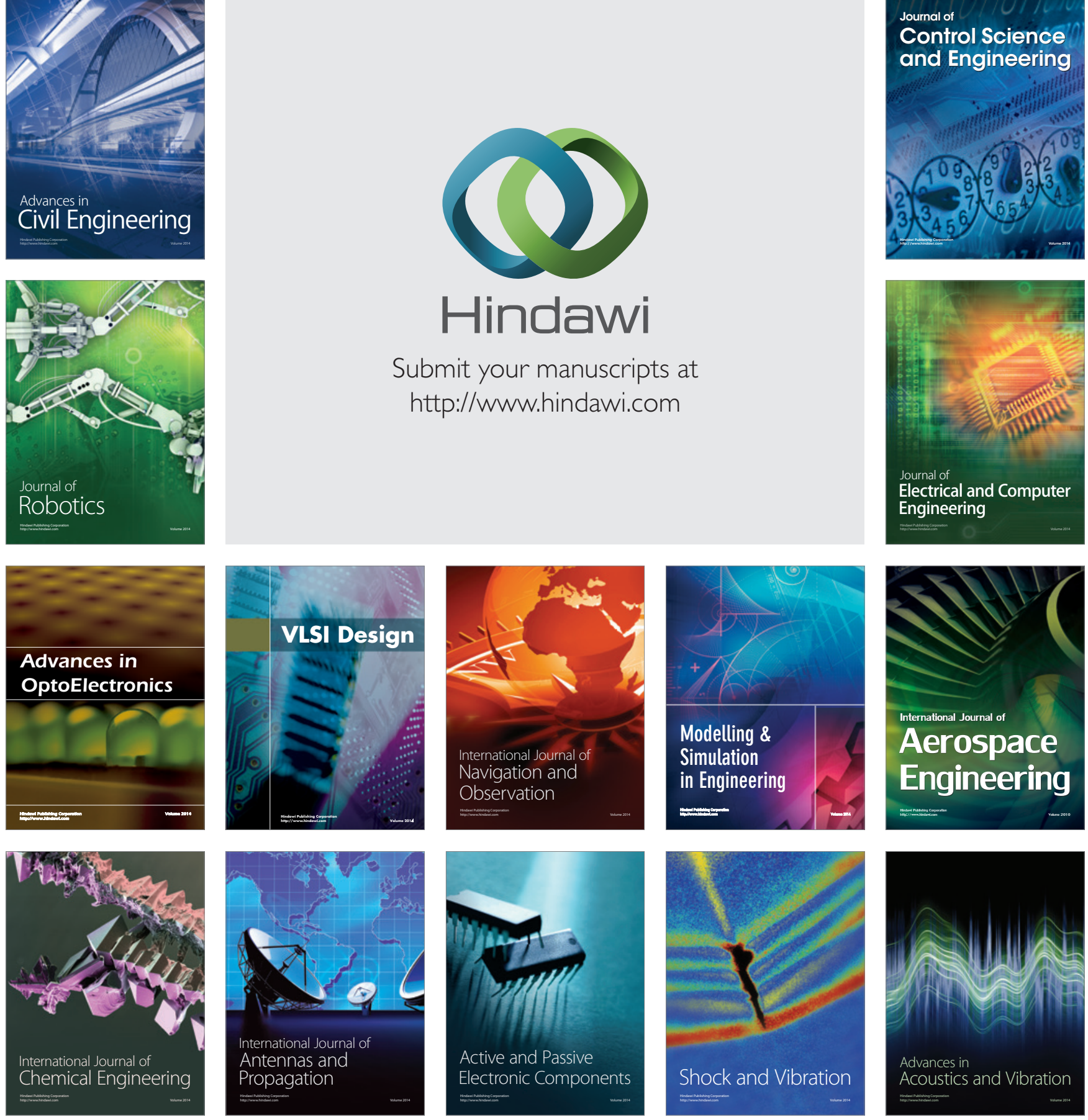